\title{
Commentary
}

About $37 \%$ of the malignant metastasis to the central nervous system is rare and it is reportedly under $3-5 \%$ of all the metastases that are resected from the brain. However, the biological reason as to why this is true is not known. There are a few other cancers, such as prostate, which are very common, but rarely spread to the brain. The treatment for solitary brain tumors is commonly carried out by surgery followed by systemic chemotherapy and radiation. This approach is found to be as effective as the treatment by stereotactic radiosurgery combined with radiotherapy.

Carcinoma of the uterine cervix is known to spread to the adjacent organs through the lymphatics to the 
pelvic and retroperitoneal lymph nodes, and/or by the hematogenous route, to other distant organs. ${ }^{[1]}$ Uterine carcinoma can be squamous cell carcinoma or adenocarcinoma, as is noticed in this case report. Brain metastasis from uterine cancer is uncommon. ${ }^{[2]}$ Very few cases of uterine cancer metastasis to the brain are reported, and it is approximately $<1 \%$ of all uterine cervix cancer cases ${ }^{[3]}$ Postoperative magnetic resonance imaging (MRI) in the present case report appears to show complete excision. There appears to be no other brain metastasis seen on the MRI.

Recurrences after long periods of remission are common, especially in less malignant phenotypes, such as the breast. Mechanistically, the explanation is not clear, other than an extremely low fraction of residual cells residing within the tumor stem cell fraction or high fraction of G0 cell population or during the process of surgery the protective wall around the formerly inactive tumor mass is disrupted allowing the cancer cells to enter the blood stream and grow as tumors in other parts of the body in due course. Some of the other cancers that rarely produce solitary metastasis to the brain include systemic malignant lymphoma, lung cancer, breast cancer, prostate cancer, melanoma, squamous cell carcinoma of the pharynx (cerebellopontine angle), renal carcinoma, malignant pleural mesothelioma, endometrial carcinoma (cerebellar metastasis), and endometrial adenocarcinoma (scalp) among others. The present case report shows solitary brain metastasis after 11 years of remission from the uterine cervical adenocarcinoma. ${ }^{[4]}$

Thus, increasing the number of primary cancer cases with brain metastasis after prolonged remission in the reported literature gives an insight into the therapy and prognosis of these patients. This would further help us to understand the metastatic behavior of these cancer cells after their primary treatment.

\section{Shivani Ponnala}

Departments of Cancer Biology and Pharmacology, University of Illinois College of Medicine at Peoria, Peoria, IL 61656, USA

\section{Address for correspondence:}

Dr. Shivani Ponnala,

Department of Cancer Biology and Pharmacology and Department of Neurosurgery, University of Illinois College of Medicine at Peoria, One Illini Drive, Peoria, IL 61656, USA. E-mail: shivani.ponnala@gmail.com

\section{References}

1. Available from: http://www.abta.org/sitefiles/pdflibrary/ABTA-Facts and Statistics 2011-FINAL.pdf [Last accessed on 2011 Oct 10].

2. Nagar YS, Shah N, Rawat S, Kataria T. Intracranial metastases from adenocarcinoma of cervix: A case report. Int J Gynecol Cancer 2005; 15:561-3.

3. Omari-Alaoui HE, Gaye PM, Kebdani T, El Ghazi E, Benjaafar N, Mansouri A, et al. Cerebellous metastases in patients with uterine cervical cancer. Two cases reports and review of the literature. Cancer Radiother 2003; 7:317-20.

4. Dadlani R, Ghosal N, Hegde AS. Solitary cerebellous metastasis after prolonged remission in a case of uterine cervical adenocarcinoma. J Neurosci Rural Pract 2012;3:185-7

\begin{tabular}{|l|l|}
\hline \multicolumn{2}{|c|}{ Access this article online } \\
\hline Quick Response Code: & \\
\hline & Website: \\
\hline
\end{tabular}

